\title{
Is adenomyosis the neglected phenotype of an endomyometrial dysfunction syndrome?
}

\author{
Ivo Brosens • Georg Kunz • Giuseppe Benagiano
}

Received: 16 November 2011 / Accepted: 24 November 2011 /Published online: 28 December 2011

(C) The Author(s) 2011. This article is published with open access at Springerlink.com

\begin{abstract}
Since the dissociation between adenomyoma and endometriosis in the 1920s and the laparoscopic progress in the diagnosis and surgery of endometriosis, the literature has been greatly focused on the disease endometriosis. The study of adenomyosis, on the other hand, has been neglected as the diagnosis remained based on hysterectomy specimens. However, since the introduction of magnetic resonance and sonographic imaging techniques in the 1980s, the myometrial junctional zone has been identified as a third uterine zone and interest in adenomyosis was renewed. This has also been the start for the interest in the role of the myometrial junctional zone dysfunction and adenomyosis in reproductive and obstetrical disorders.
\end{abstract}

Keywords Adenomyosis · Endometriosis

\section{Introduction}

Endometriosis and adenomyosis are both defined by the presence of ectopic endometrial-like tissue, respectively outside the uterus and in the myometrium. Although both disorders were described under the common name of "adenomyoma" by

I. Brosens $(\bowtie)$

Leuven Institute for Fertility and Embryology,

Leuven, Belgium

e-mail: ivo.brosens@med.kuleuven.be

G. Kunz

Department of Obstetrics and Gynaecology, St-Johannes-Hospital,

Dortmund, Germany

G. Benagiano

Department of Gynaecology, Obstetrics and Urology,

University of Rome,

Sapienza, Italy early authors [1] and extensively studied by Cullen [2] as one condition, today they are considered two different diseases. The dissociation practiced in the 1920s by Frankl [3] and Sampson [4] is largely a consequence of the limitations in diagnostic tools that have existed up to the mid-1980s. Today, new insights in the structural and functional characteristics of the endometrium and myometrial junctional zone (the "endomyometrium"), in women with adenomyosis and endometriosis, provide increasing evidence that they are frequently associated and both are characterized by an aberrant function of the endomyometrium. Data indicating that they could well represent two phenotypes of a similar endomyometrial dysfunction syndrome rather than two different diseases have been accumulated - thanks to current imaging techniques, functional, and molecular investigations. In addition, evidence has also been gathered that the endomyometrial dysfunction has major repercussion on reproductive functions. The present review first describes the history and clinical background of the two anomalies; subsequently, it outlines reproductive dysfunctions existing in both disorders and, finally, tries to define clinical features of the syndrome.

\section{Historical diagnostic bias}

Glandular inclusions in a number of pelvic sites were identified during the second part of the nineteenth century; their presence created a controversy that continued for decades. It was Cullen [5] that, in 1896, unequivocally stated that these pelvic inclusions were derived from the "mucous membrane of the uterus". Then, in 1920, he provided a detailed description of a number of ectopic sites where the inclusions could be present, including the outer myometrium, the rectovaginal septum, uterine ligaments, the muscular wall of bladder and bowel, and other abdominal structures and coined the lesions 
adenomyomas [2, 6] (Fig. 1). In 1927, Sampson [4] formulated the hypothesis that "peritoneal endometriosis" was "due to the menstrual dissemination of endometrial tissue into the peritoneal cavity". From there on, uterine adenomyosis, which could not be explained by regurgitation of menstrual elements, was coined in 1925 by Frankl [3] adenomyosis; and endometriosis, with its separate pathogenesis, became widely regarded as a disease entity distinct from adenomyosis.

Although already in the 40s, Novak and de Lima [7] linked again adenomyosis and endometriosis, stating "one cannot resist the feeling that there is some common denominator between endometrial hyperplasia and adenomyosis, and possibly also pelvic endometriosis", it was only over the last two decades that the search for the underlying link between endometriosis and adenomyosis received a major booster. The development that provided the first important lead was the introduction of magnetic resonance (MR) imaging; using this technique, the "myometrial junctional zone" (JZ) was identified as a functional uterine zone to form with the endometrium

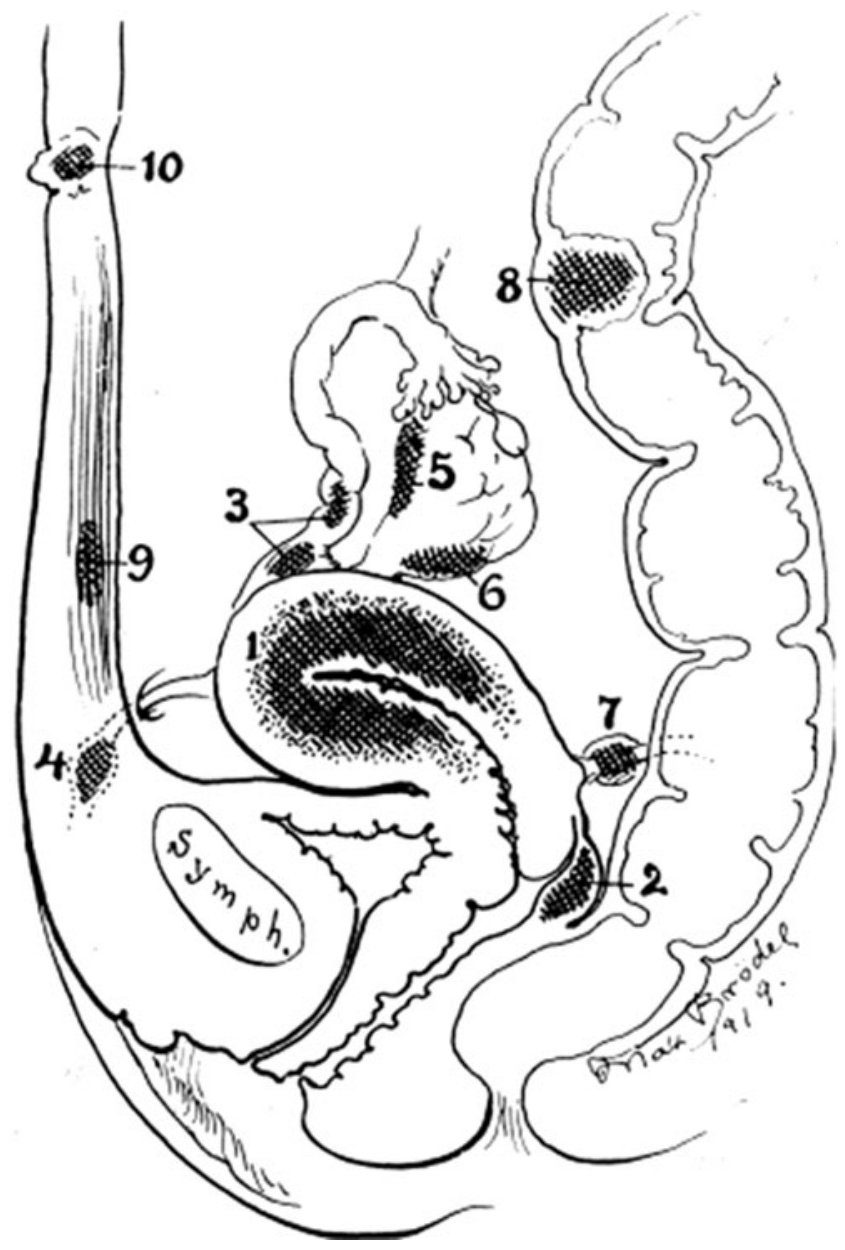

Fig. 1 Diagram showing localizations of ectopic endometrial tissue (indicated as adenomyomas): 1 , uterine wall; 2 , rectovaginal septum; 3 , fallopian tubes; 4 , round ligament; 5 , hilum of ovary; 6 , utero-ovarian ligament; 8 , colon; 9, musculus rectus; 10, umbilicus. From Cullen [2] with permission the endomyometrium unit [8]. Shortly after, numerous molecular studies showed that the endometrium in endometriosis and adenomyosis is characterized by impaired cellular responses to ovarian sex steroids [9], as well as by a number of other metabolic aberrations.

Notwithstanding, these important developments, a major stumbling block remained since the histological diagnosis of endometriosis is based on a simple biopsy obtained at the time of laparoscopy or laparotomy, while the diagnosis of adenomyosis traditionally required a hysterectomy specimen. Indeed, to this day, almost all studies involving adenomyosis have been carried out on uteri removed at surgery. This has major scientific consequences. First, according to Scopus, the number of publications in 2011 on endometriosis is 22,730 and on adenomyosis 2,368, a tenfold difference. Secondly, endometriosis is frequently diagnosed in its early stage by the presence of variable or scarred peritoneal endometriosis, while adenomyosis is rarely diagnosed during the early reproductive life, its identification being made only after the appearance, in older women, of symptoms of abnormal menstrual bleeding and/or dysmenorrhea, severe enough to necessitate hysterectomy. Consequently, endometriosis risks to be overdiagnosed, while adenomyosis is neglected or overlooked.

\section{The endomyometrium link}

\section{Aberrant eutopic endometrium}

Although histologically normal, a series of biochemical evaluations of the endometrium in women with endometriosis and adenomyosis has, already in the late 1990s, revealed profound disturbances. The eutopic endometrium in women with these conditions is characterized by a large range of abnormalities including alterations in immune cell population [10], aberrant expression of aromatase [11], dysregulation of IL-6 production [12], impaired temporal expression of beta3integrin [13], and HOX genes [14] and reduced spontaneous apoptosis [15]. During the last decade, many more molecules and functions have been found to be altered in endometriosis [16] and adenomyosis [17].

An additional problem in any attempt at comparatively analyzing endometrial abnormalities is given by the diagnostic modality utilized for endometriosis. On one hand, endometrial biopsies are easily obtained at the time of laparoscopy for the diagnosis of endometriosis; on the other, the contemporary presence of adenomyosis cannot be excluded in the vast majority of these studies due to the absence of a hysterectomy specimen or imaging scan. As a consequence, most studies on the endometrium in endometriosis have failed to exclude the presence of adenomyosis, whereas, as it will be detailed below, several studies - although disagreeing on the percentage - have shown that the two conditions coexist in a 
significant proportion of cases; therefore, a major bias persists in the biochemical evaluation of the eutopic endometrium in women with endometriosis when adenomyosis is not excluded.

\section{Aberrant myometrial junctional zone}

Since the junctional zone myometrium started to be investigated by MR imaging, every abnormality observed has been described as indicative of the presence of endometrium in the outer myometrium or adenomyosis sensu stricto. In fact, however, distinction should be made between junction zone abnormalities and adenomyosis. Tocci et al. [18] have now proposed that the "endometrial-subendometrial myometrium unit (or junctional zone myometrium) disruption disease" be considered as a new entity distinguished from adenomyosis. The diagnosis of which is feasible and straightforward on imaging. This condition is expressed mainly by a pathological thickening or abnormality of the subendometrial myometrium (myometrial halo or junctional zone). Clearly, there is a need for uniform terminology and classification [19] and until this is agreed upon, confusion will persist.

Although it is generally accepted that adenomyosis develops as a result of downward growth of the endometrium into the myometrium, MR imaging studies suggest that smooth muscle proliferation and hyperplasia in the junctional zone may precede the outgrowth of endometrial cells [20]. Leyendecker et al. [21] speculated that disruption of the specific uterine microenvironment in the basal endometrium may explain the structural and functional abnormalities of the junctional zone, such as hyperperistalsis, dysperistalsis, and inordinate smooth muscle proliferation associated with endometriosis and adenomyosis.

In a case-control-blinded comparison, Mehasseb et al. [22] studied the morphometric features of the myometrium in uteri from pre- and postmenopausal women with and without uterine adenomyosis as the sole pathology. Uteri were also divided according to the phase of the cycle and examined using immunohistochemistry and image analysis. The authors found clear differences between the inner myometrium and the outer myometrium, but the transition is gradual, with no distinct "zonation". Adenomyosis is characterized by reduced cell density and increased nuclear size and features of hyperplasia and hypertrophy that are not confined to the inner myometrium.

In a previous study, the same authors [23] found that smooth muscle cells from uteri with adenomyosis are ultrastructurally different from smooth muscle cells of normal uteri. They suggested a possible effect of these distinct features on myometrial contractility, together with hypertrophy.

Asssociation between endometriosis and adenomyosis

Using MR imaging, Kennedy et al. [24] found a high correlation between the presence of endometriosis and of adenomyosis in first degree relatives, suggesting that both are phenotypes of a single disorder rather that two distinct disease entities. Pelvic endometriosis, especially in severe stages, is strongly associated with JZ thickening [25-27]. In 2005, Kunz et al. [26] tested the hypothesis of a strong association between endometriosis and adenomyosis with adenomyosis playing a role in causing infertility in women with endometriosis. Using magnetic resonance imaging, they found that the posterior junctional zone (PJZ) was significantly thicker in women with endometriosis than in those without the disease. The MR imaging method as described in their previous publication [28] allowed them to identify alterations of the junctional zone that were interpreted as signs of focal and diffuse adenomyosis, respectively, according to the guidelines provided by Reinhold et al. [29]. A threshold value of more than $10 \mathrm{~mm}$ was chosen, above which with additional signs up to $12 \mathrm{~mm}$, diffuse adenomyosis was assumed. There was a positive correlation of the diameter of the PJZ with the stage of the disease and the age of the patients. The prevalence of adenomyotic lesions in all 160 women with endometriosis was $79 \%$. In women with endometriosis below an age of 36 years and fertile partners, the prevalence of adenomyosis was 90\% $(p<0.01)$. Kunz et al. [26] concluded that with a prevalence of up to $90 \%$, uterine adenomyosis is significantly associated with pelvic endometriosis and constitutes an important factor of sterility in endometriosis presumably by impairing uterine sperm transport.

With respect to the effect of uterine contractions as creating thickening of the JZ, Kunz et al. [26] clarified that these contractions only exceeded those of healthy women during the late menstrual and early follicular phase, whereas the study was not restricted to these phases of the menstrual cycle. Moreover, these contractions were never confined to the posterior wall myometrium or prevailed as compared to the anterior wall contractions, hence always comprising the anterior as well as the posterior uterine myometrium within one contraction wave. But only the PJZ showed this highly significant difference in relation to the incidence of adenomyosis. They concluded that a possible bias with uterine wall contractions influencing the findings obtained from the 227 patients cannot be absolutely excluded, but appears to be negligible.

Traditionally, the diagnosis of adenomyosis is obtained from women in their fourth to fifth decade of life. However, recent $\mathrm{MR}$ imaging data suggest that adenomyosis may develop much sooner in life, particularly in women with endometriosis. In order to test this hypothesis, Kunz et al. [30] performed MR imaging of the uterus in 227 women with and without endometriosis and related the results to the age of the subjects (age groups, 17-24, 25-29, 30-34, and >34 years). The study revealed that the process of development of adenomyosis, represented by an increased diameter of the dorsal junctional zone of the uterus (the imaging feature indicative of 
the invasion of basal endometrium into the junctional zone), had already commenced early in the third decade of life and progressed steadily during the fourth decade in women with endometriosis. Women without endometriosis showed almost no signs of adenomyosis up to the age of 34 years. Surprisingly, in both groups of women, a parallel marked increase in the incidence of adenomyosis could be observed beyond the age of 34 years, thus representing a common phenomenon in the age-related pathophysiological continuum of adenomyosis.

Furthermore, in a prospective study of 70 patients presenting with severe dysmenorrhea, Kissler et al. [31] found that dysmenorrhea of long duration in patients who have had endometriosis for over a threshold value of 11 years is significantly related to adenomyosis. Hence, evaluation of adenomyosis using MRI should become a standard procedure in cases of severe dysmenorrhea associated with endometriosis and, if of long duration, this symptom should always pose a suspicion of adenomyosis. Finally, in 2010, Mijatovic et al. [32], in a retrospective study of 74 infertile patients with surgically proven endometriosis ( $90.4 \%$ of them with endometriosis rASRM stages III-IV), found also the presence of adenomyosis in $27 \%$ of them, predominantly located in the posterior wall of the uterus.

Recently Larsen et al. [33] correlated the degree of adenomyotic infiltration with the degree of infiltration and stage of endometriosis. MR imaging of the uterus was performed in 153 women with suspected deeply infiltrating endometriosis and planned surgery, and in a reference group of 129 women without endometriosis, verified during hysterectomy. Diagnosis of adenomyosis at MR imaging was based on optimal criteria derived from the hysterectomy control group, while the stage of endometriosis (rAFS stage) was determined during surgery. The authors found that in the group of women with endometriosis, $34.6 \%$ had adenomyosis compared with $19.4 \%$ in the reference group $(p<0.05)$. More women with endometriosis (39.9\%) had an irregular JZ compared to $22.5 \%$ in the reference group $(p<0.01)$. Among women with severe endometriosis (AFS stage IV), $42.8 \%$ had adenomyosis compared to $29.4 \%$ in the women with other stages of endometriosis (AFS stages I + II + III) $(p=0.10)$. More women with severe endometriosis (AFS stage IV) had deeper wall invasion of adenomyosis $(p>0.05)$, but the presence of deep infiltrative rectovaginal endometriosis and the size of infiltration were not correlated to adenomyosis or to the depth of infiltration of adenomyosis. The authors concluded that, based on a systematic evaluation of the junctional zone by MR imaging, severe symptomatic endometriosis is in one third of the cases associated with uterine adenomyosis. For some reason, however, "deep endometriosis" and "deep adenomyosis" correspond only to a limited degree.

\section{The potential role of endomyometrial dysfunction in reproductive and obstetrical disorders}

In the normal human pregnancy, the remodeling of the spiral arteries involves both their myometrial and decidual segments. There is growing evidence that a pre-pregnancy disorder of the myometrial junctional zone is at the basis of defective deep placentation which, in turn, is associated with major reproductive and obstetrical complications [34]. Comparative studies on placentation show that the human is not unique among primates in showing deep trophoblast invasion [35], although recent investigations indicate that the baboon, with no appreciable trophoblast invasion of the myometrium is not an appropriate model for the study of either endometriosis [36] or preeclampsia [35]. On the other hand, there are case reports showing that preeclampsia may occur in chimpanzees and gorillas where trophoblast invasion and spiral artery remodeling are basically similar to the human $[37,38]$.

Today, the investigation of reproductive outcomes in young women with junctional zone abnormalities and/or adenomyosis has become possible - thanks to MR imaging and ultrasonography; using these techniques, an improved and clinically useful correlation with histology-based diagnosis for adenomyosis has been accomplished $[39,40]$ - thanks also to the analysis of the myometrial junctional zone. The importance of myometrial junctional zone research in reproductive medicine is frequently underestimated: indeed, the inner myometrium is the critical zone for the adequate transformation of spiral arteries supplying maternal blood to intervillous spaces of the placenta and a defective transformation of myometrial spiral arteries in the placental bed has been associated with major obstetrical syndromes [41].

The disappearance of the distinct myometrial zonal anatomy very early during pregnancy on MR imaging has also been observed in a unique case report by Turnbull et al. [42]. This may correspond to the early decidualization process of the myometrial spiral arteries in the placental bed well before the zone is colonized by intravascular and interstitial trophoblast $[43,44]$.

Impaired follicular function

A recent MR imaging study by Kunz and Beil [45] suggests that the uterine junctional zone may be of significance with respect to the early process of human reproduction. In 113 infertile women, the diameter of the anterior and posterior JZ myometrium was documented by MR imaging prior to the use of in vitro fertilization (IVF)/intracytoplasmic sperm injection (ICSI). Each group of women was also subdivided according to the presence [metaphase I (MI)/germinal vesicle $(\mathrm{GV})>0 \%$ ] or absence $(\mathrm{MI} / \mathrm{GV}=0 \%)$ of $\mathrm{MI}$ and $\mathrm{GV}$ oocytes. The MRI measurements were related to these and further 
parameters like expansion of the cumulus oophorus, pronuclei stages, embryo morphology, and pregnancy rates. The authors found that in all women with $\mathrm{MI} / \mathrm{GV}=0 \%$, the $\mathrm{JZ}$ myometrium was significantly enlarged $(p<0.004)$ and the clinical pregnancy rate was significantly lower $(p<0.025)$ compared to that of women with $\mathrm{MI} / \mathrm{GV}>0 \%$. The authors suggested that the JZ, as documented by MR imaging, might interfere with follicular function.

Implantation failure and miscarriage

In a prospective clinical-imaging study including 152 patients, Maubon et al. [46] have explored the influence of the uterine junctional zone thickness (as measured on pelvic MR imaging), on implantation rates during IVF. They measured average and maximal junctional zone thickness values and implantation outcomes were correlated with these values and with infertility subtypes (endometriosis, tubal, dysovulation, male, and unexplained). A junctional zone thickness increase was significantly correlated with implantation failure at IVF: implantation failure rate was $95.8 \%$ for patients with an average junctional zone greater than $7 \mathrm{~mm}$ and a maximal junctional zone greater than $10 \mathrm{~mm}$, versus $37.5 \%$ in other patient groups $(p<0.0001)$, independently from cause of infertility or patients' age. The authors concluded that a pelvic MR scan showing a thickened uterine junctional zone has a negative predictive factor for embryo implantation after IVF.

Tremellen and Russell [47] reported that in four women who had previously undergone multiple unsuccessful in vitro fertilization attempts, inactivation of adenomyosis by an ultra-long pituitary downregulation regime, promptly resulted in successful pregnancy for all four women. In women with endometriosis, Mijatovic et al. [32] found no significant differences for any of the IVF/ICSI outcomes between women with and without concomitant adenomyosis after pretreatment with GnRH agonist for 3 to 26 months. Clearly, further prospective controlled studies are required to clarify the impact of adenomyosis on implantation following IVF.

Martinez-Conejero et al. [48] evaluated the effect of adenomyosis on endometrial gene expression and reproductive outcome in an oocyte donation program. The clinical and molecular data indicate that implantation is not affected by adenomyosis, but higher rates of miscarriage were associated with this condition leading to lower term pregnancies.

Major obstetrical syndromes

On the basis of pre-pregnancy imaging, Juang et al. [49] reported that adenomyosis is an important risk factor for spontaneous preterm delivery. Other recent studies have shown that endometriosis is associated with preterm delivery and other major obstetrical syndromes, such as small for gestation age in the absence of hypertension, preterm delivery, and obstetrical abnormal bleeding [50-52]. In evaluating the significance of these investigations, once again the question arises whether adenomyosis may not be a critical confounding factor in the endometriosis studies as long as adenomyosis or junctional zone hyperplasia is not systematically excluded by imaging scan. In addition, advanced maternal age is not only an important confounding factor for the likelihood of pregnancy complications, but is also associated with structural changes in JZ anatomy as demonstrated by Kunz et al. [30]. Indeed, observational studies suggest that perturbations in JZ structure or functions prior to conception predispose towards impaired deep trophoblast invasion that underpins a spectrum of obstetrical complications, including preterm birth [53-55]

\section{Conclusions}

While the link between endometriosis and endometriosis has been puzzling, the role of the myometrial junctional zone appears in both conditions to be associated with several pathologically and clinically recognizable features. Therefore, the presence of either endometriosis or adenomyosis should alert the physician to the possible presence of the other. Both tend to have a range of possible etiologies that could create a set of reproductive and obstetrical disorders. Although the reason that they occur together has not yet been discovered, they have several molecular aberrations of the endometrium and myometrial junction zone in common. As a consequence, these features can justify the introduction of the endomyometrium dysfunctional syndrome.

Finally, there are several practical implications for research and clinical management. It is clear that, from now on, biochemical and clinical studies of the eutopic endometrium in women with endometriosis need to document the presence or absence of myometrial junctional zone abnormalities and uterine adenomyosis.

Conflicts of interest The authors report no conflicts of interest. The authors alone are responsible for the content and writing of the paper.

Open Access This article is distributed under the terms of the Creative Commons Attribution Noncommercial License which permits any noncommercial use, distribution, and reproduction in any medium, provided the original author(s) and source are credited.

\section{References}

1. Benagiano G, Brosens I (2011) Who identified endometriosis? Fertil Steril 95(1):13-16 
2. Cullen TS (1920) The distribution of adenomyomata containing uterine mucosa. Arch Surg 1:215-283

3. Frank1 O (1925) Adenomyosis uteri. Am J Obstet Gynecol 10:680-684

4. Sampson JA (1927) Peritoneal endometriosis due to the menstrual dissemination of endometrial tissue into the peritoneal cavity. Am J Obstet Gynecol 14:422-469

5. Cullen TS (1896) Adeno-myoma Uteri diffusum benignum. Johns Hopkins Hosp Rep 6:133

6. Kuligowska E, Deeds L 3rd, Lu K 3rd (2005) Pelvic pain: overlooked and underdiagnosed gynecologic conditions. Radiographics 25(1):3-20

7. Novak E, de Lima OA (1948) A correlative study of adenomyosis and pelvic endometriosis, with special reference to the hormonal reaction of ectopic endometrium. Am J Obstet Gynecol 56:634 644

8. Hricak H, Alpers C, Crooks LE, Sheldon PE (1983) Magnetic resonance imaging of the female pelvis: initial experience. Am J Roentgen 141(6):1119-1128

9. Brosens IA, Brosens JJ (2000) Endometriosis. Eur J Obstet Gynecol Reprod Biol 90(2):159-164

10. Bulmer JN, Jones RK, Searle RF (1998) Intraepithelial leukocytes in endometriosis and adenomyosis: comparison of eutopic and ectopic endometrium with normal endometrium. Hum Reprod 13 (10):2910-2915

11. Noble LS, Takayama K, Zeitoun KM, Putman JM, Johns DA, Hinshelwood MM, Agarwal VR, Zhao Y, Carr BR, Bulun SE (1997) Prostaglandin E2 stimulates aromatase expression in endometriosis-derived stromal cells. J Clin Endocrinol Metab 82 (2):600-606

12. Tseng JF, Ryan IP, Milam TD, Murai JT, Schriock ED, Landers DV, Taylor RN (1996) Interleukin-6 secretion in vitro is upregulated in ectopic and eutopic endometrial stromal cells from women with endometriosis. J Clin Endocrinol Metab 81(3):1118 1122

13. Ota H, Tanaka T (1997) Integrin adhesion molecules in the endometrial glandular epithelium in patients with endometriosis or adenomyosis. J Obstet Gynaecol Res 23(5):485-491

14. Taylor HS, Bagot C, Kardana A, Olive D, Arici A (1999) HOX gene expression is altered in the endometrium of women with endometriosis. Hum Reprod 14(5):1328-1331

15. Dmowski WP, Steele RW, Baker GF (1981) Deficient cellular immunity in endometriosis. Am J Obstet Gynecol 141(4):377-383

16. Carvalho L, Podgaec S, Bellodi-Privato M, Falcone T, Abrão MS (2011) Role of eutopic endometrium in pelvic endometriosis. J Minim Invasive Gynecol 18(4):419-427

17. Benagiano G, Brosens I (2011) Adenomyosis and endometriosis have a common origin. J Obstet Gynecol India 61:27-31

18. Tocci A, Greco E, Ubaldi FM (2008) Adenomyosis and 'endometrialsubendometrial myometrium unit disruption disease' are two different entities. Reprod BioMed Online 17(2):285-291

19. Gordts S, Brosens JJ, Fusi L, Benagiano G, Brosens I (2008) Uterine adenomyosis: a need for uniform terminology and consensus classification. Reprod Biomed Online 17(2):244-248

20. Brosens JJ, de Souza NM, Barker FG (1995) Uterine junctional zone: function and disease. Lancet 346(8974):558-560

21. Leyendecker G, Kunz G, Noe M, Herbertz M, Mall G (1998) Endometriosis: a dysfunction and disease of the archimetra. Hum Reprod Update 4(5):752-762

22. Mehasseb MK, Bell SC, Brown L, Pringle JH, Habiba M (2011) Phenotypic characterisation of the inner and outer myometrium in normal and adenomyotic uteri. Gynecol Obstet Invest 71 (4):217-224

23. Mehasseb MK, Bell SC, Pringle JH, Habiba MA (2010) Uterine adenomyosis is associated with ultrastructural features of altered contractility in the inner myometrium. Fertil Steril 93(7):2130-2136
24. Kennedy S, Hadfield R, Westbrook C, Weeks DE, Barlow D, Golding S (1998) Magnetic resonance imaging to assess familial risk in relatives of women with endometriosis. Lancet 352(9138): 1440-1441

25. Devlieger R, D'Hooghe T, Timmerman D (2003) Uterine adenomyosis in the infertility clinic. Hum Reprod Update 9(2):139-147

26. Kunz G, Beil D, Huppert P, Noe M, Kissler S, Leyendecker G (2005) Adenomyosis in endometriosis - Prevalence and impact on fertility. Evidence from magnetic resonance imaging. Hum Reprod 20(8):2309-2316

27. Landi S, Mereu L, Pontrelli G, Stepniewska A, Romano L, Tateo S, Dorizzi C, Minelli L (2008) The influence of adenomyosis in patients laparoscopically treated for deep endometriosis. J Minim Invasive Gynecol 15(5):566-570

28. Kunz B, Beil D, Huppert P, Leyendecker G (2000) Structural abnormalities of the uterine wall in women with endometriosis and infertility visualized by vaginal sonography and magnetic resonance imaging. Hum Reprod 15(1):76-82

29. Reinhold C, Tafazoli F, Mehio A, Wang L, Atri M, Siegelman ES, Rohoman L (1999) Uterine adenomyosis: endovaginal US and MR imaging features with histopathologic correlation. Radiographics 19:S147-S160

30. Kunz G, Herbertz M, Beil D, Huppert G, Leyendecker G (2007) Adenomyosis as a disorder of the early and late human reproductive period. Reprod Biomed Online 15(6):681-685

31. Kissler S, Zangos S, Kohl J, Wiegratz I, Rody A, Gätje R, Vogl TJ, Kunz G, Leyendecker G, Kaufmann M (2008) Duration of dysmenorrhoea and extent of adenomyosis visualised by magnetic resonance imaging. Eur J Obstet Gynecol Reprod Biol 137(2):204-209

32. Mijatovic V, Florijn E, Halim N, Schats R, Hompes P (2010) Adenomyosis has no adverse effects on IVF/ICSI outcomes in women with endometriosis treated with long-term pituitary down-regulation before IVF/ICSI2010. Eur J Obstet Gynecol Reprod Biol 151(1):62-65

33. Larsen SB, Lundorf E, Forman A, Dueholm M (2011) Adenomyosis and junctional zone changes in patients with endometriosis. Eur $\mathrm{J}$ Obstet Gynecol Reprod Biol 157(2):206-211

34. Brosens I, Derwig I, Brosens J, Fusi L, Benagiano G, Pijnenborg R (2010) The enigmatic uterine junctional zone: the missing link between reproductive disorders and major obstetrical disorders? Hum Reprod 25(3):569-574

35. Carter AM, Pijnenborg R (2011) Evolution of invasive placentation with special reference to non-human primates. Best Pract Res Clin Obstet Gynaecol 25(3):249-257

36. Dehoux J-P, Defere S, Squifflet J, Donnez O, Polet R, Mestdagt M, Foidart J-M, Van Langendonckt A, Donnez J (2011) Is the baboon model appropriate for endometriosis studies? Fertil Steril 96 (3):728-733.e3

37. Pijnenborg R, Vercruysse L, Carter AM (2011) Deep trophoblast invasion and spiral artery remodelling in the placental bed of the lowland gorilla. Placenta 32(8):586-591

38. Pijnenborg R, Vercruysse L, Carter AM (2011) Deep trophoblast invasion and spiral artery remodelling in the placental bed of the chimpanzee. Placenta 32(4):400-408

39. Bazot M, Darai E, Clement de Givry S, Boudghene F, Uzan S, Le Blanche AF (2003) Fast breath-hold T2-weighted MR imaging reduces interobserver variability in the diagnosis of adenomyosis. Am J Roentgenol 180(5):1291-1296

40. Lone FW, Balogun M, Khan KS (2006) Adenomyosis: not such an elusive diagnosis any longer. J Obstet Gynaecol 26(3):225-228

41. Brosens I, Pijnenborg R, Vercruysse L, Romero R (2011) The "Great Obstetrical Syndromes" are associated with disorders of deep placentation. Am J Obstet Gynecol 204:193-201

42. Turnbull LW, Manton DJ, Horsman A, Killick SR (1995) Magnetic resonance imaging changes in uterine zonal anatomy during a conception cycle. Brit J Obstet Gynaecol 102(4):330-331 
43. Brosens IA (1977) Morphological changes in the utero-placental bed in pregnancy hypertension. Clin Obstet Gynaecol 4(3): 573-593

44. Pijnenborg R, Bland JM, Robertson WB, Brosens I (1983) Uteroplacental arterial changes related to interstitial trophoblast migration in early human pregnancy. Placenta 4(4):397-414

45. Kunz G, Kunz G, Beil D (2010) Characterization of the uterine junctional zone prior to IVF/ICSI: an observational study (2010). Archiv Gynecol Obstet 281(5):945-953

46. Maubon A, Faury A, Kapella M, Pouquet M, Piver P (2010) Uterine junctional zone at magnetic resonance imaging: a predictor of in vitro fertilization implantation failure. J Obstet Gynaecol Res 36(3):611-618

47. Tremellen K, Russell P (2011) Adenomyosis is a potential cause of recurrent implantation failure during IVF treatment. Aust NZ J Obstet Gynaecol 51(3):280-283

48. Martínez-Conejero JA, Morgan M, Montesinos M, Fortuño S, Meseguer M, Simón C, Horcajadas JA, Pellicer A (2011) Adenomyosis does not affect implantation, but is associated with miscarriage in patients undergoing oocyte donation. Fertil Steril 96(4):943-950.e1

49. Juang C-M, Chou P, Yen M-S, Twu N-F, Horng H-C, Hsu W-L (2007) Adenomyosis and risk of preterm delivery. Brit J Obstet Gynaecol 114(2):165-169
50. Fernando S, Breheny S, Jaques AM, Halliday JL, Baker G, Healy D (2009) Preterm birth, ovarian endometriomata, and assisted reproduction technologies. Fertil Steril 91(2):325-330

51. Stephansson O, Kieler H, Granath F, Falconer H (2009) Endometriosis, assisted reproduction technology, and risk of adverse pregnancy outcome. Hum Reprod 24(9):2341-2347

52. Healy DL, Breheny S, Halliday J, Jaques A, Rushford D, Garrett C, Talbot JM, Baker HWG (2010) Prevalence and risk factors for obstetric haemorrhage in 6730 singleton births after assisted reproductive technology in Victoria Australia. Hum Reprod 25(1):265-274

53. Arias F, Victoria A, Cho K, Kraus F (1997) Placental histology and clinical characteristics of patients with preterm premature rupture of membranes. Obstet Gynecol 89(2):265-271

54. Kim YM, Chaiworapongsa T, Gomez R, Bujold E, Yoon BH, Rotmensch S, Thaler HT, Romero R (2002) Failure of physiologic transformation of the spiral arteries in the placental bed in preterm premature rupture of membranes. Am J Obstet Gynecol 187 (5):1137-1142

55. Kim YM, Bujold E, Chaiworapongsa T, Gomez R, Yoon BH, Thaler HT, Rotmensch S, Romero R (2003) Failure of physiologic transformation of the spiral arteries in patients with preterm labor and intact membranes. Am J Obstet Gynecol 189(4):1063-1069 